Presented at: American Academy of Neurology Annual Meeting; April 21-27, 2018, Los Angeles, California, USA.

Funding Acknowledgements: This study was supported by Teva Pharmaceuticals, Petach Tikva, Israel.

46

\section{Confirmed Safety of Deutetrabenazine for Tardive Dyskinesia in a 2-Year Open-label Extension Study}

Hubert H. Fernandez, $\mathrm{MD}^{\prime}$; David Stamler, $\mathrm{MD}^{2}$; Mat D. Davis, PhD ; Stewart A. Factor, DO ; Robert A. Hauser, MD, MBA ${ }^{5}$; Joohi Jimenez-Shahed, $M D^{6}$; William G. Ondo, $M D^{7}$; L. Fredrik Jarskog, $M D^{8}$; Scott W. Woods, MD ${ }^{9}$; Mark S. LeDoux, MD, PhD ${ }^{10}$; David R. Shprecher, DO, $M S^{11}$; and Karen E. Anderson, $M D^{12}$

${ }^{1}$ Cleveland Clinic, Center for Neurological Restoration, Cleveland, Ohio, USA

2 Teva Pharmaceuticals, La Jolla, California, USA

${ }^{3}$ Teva Pharmaceuticals, Frazer, Pennsylvania, USA

${ }^{4}$ Emory University, Atlanta, Georgia, USA

${ }^{5}$ University of South Florida Parkinson's Disease and Movement Disorders Center, Tampa, Florida, USA

${ }^{6}$ Baylor College of Medicine, Houston, Texas, USA

${ }^{7}$ Methodist Neurological Institute, Houston, Texas, USA; Weill Cornell Medical College, New York, New York, USA

${ }^{8}$ University of North Carolina School of Medicine, Chapel Hill, North Carolina, USA

${ }^{9}$ Yale School of Medicine, New Haven, Connecticut, USA

${ }^{10}$ University of Tennessee Health Science Center, Memphis, Tennessee, USA

${ }^{11}$ University of Utah, Salt Lake City, Utah, USA; Banner Sun Health Research Institute, Sun City, Arizona, USA

${ }^{12}$ Georgetown University, Washington, District of Columbia, USA

ABSTRACT: Study Objective: To evaluate the long-term safety and tolerability of deutetrabenazine in patients with tardive dyskinesia (TD) at 2 years.

BACKGROUND: In the 12-week ARM-TD and AIM-TD studies, deutetrabenazine showed clinically significant improvements in Abnormal Involuntary Movement Scale scores compared with placebo, and there were low rates of overall adverse events (AEs) and discontinuations associated with deutetrabenazine.

METHOD: Patients who completed ARM-TD or AIM-TD were included in this open-label, single-arm extension study, in which all patients restarted/started deutetrabenazine $12 \mathrm{mg} /$ day, titrating up to a maximum total daily dose of $48 \mathrm{mg} /$ day based on dyskinesia control and tolerability. The study comprised a 6-week titration period and a long-term maintenance phase. Safety measures included incidence of AEs, serious AEs (SAEs) and AEs leading to withdrawal, dose reduction, or dose suspension. Exposure-adjusted incidence rates (EAIRs; incidence/patient-years) were used to compare $\mathrm{AE}$ frequencies for long-term treatment with those for short-term treatment (ARM-TD and AIM-TD). This analysis reports results up to 2 years (Week 106).

RESULTS: 343 patients were enrolled (111 patients received placebo in the parent study and 232 received deutetrabenazine). There were 331.4 patient-years of exposure in this analysis. Through Week 106, EAIRs of AEs were comparable to or lower than those observed with short-term deutetrabenazine and placebo, including AEs of interest (akathisia/restlessness [long-term EAIR: 0.02; short-term EAIR range: 0-0.25], anxiety [0.09; $0.13-0.21]$, depression $[0.09 ; 0.04-0.13]$, diarrhea [0.06; 0.06-0.34], parkinsonism [0.01; 0-0.08], somnolence/sedation [0.09; 0.06-0.81], and suicidality [0.02; $0-0.13]$ ). The frequency of SAEs (EAIR 0.15) was similar to those observed with short-term placebo $(0.33)$ and deutetrabenazine (range 0.06-0.33) treatment. AEs leading to withdrawal (0.08), dose reduction (0.17), and dose suspension $(0.06)$ were uncommon.

CONCLUSIONS: These results confirm the safety outcomes seen in the ARM-TD and AIM-TD parent studies, demonstrating that deutetrabenazine is well tolerated for long-term use in TD patients.

Presented at: American Academy of Neurology Annual Meeting; April 21-27, 2018, Los Angeles, California, USA Funding Acknowledgements: Funding: This study was supported by Teva Pharmaceuticals, Petach Tikva, Israel

47

\section{Sustained Functional Recovery and Symptom Remission After Maintenance Treatment with Aripiprazole Once-Monthly for Patients with Bipolar I Disorder}

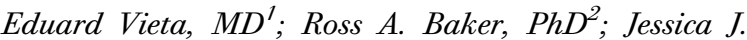
Madera, $\mathrm{MD}^{3}$; Peter Zhang, PhD ${ }^{4}$; Pedro Such, $\mathrm{MD}^{5}$. Maxine Chen, $P h D^{6}$; and Joseph Calabrese, $M D^{7}$

${ }^{1}$ Chair, Department of Psychiatry and Psychology, Hospital Clinic, Bipolar Unit, Institute of Neuroscience, University of Barcelona, IDIBAPS, CIBERSAM, Barcelona, Catalonia, Spain

${ }^{2}$ Director, Global Medical Affairs, Otsuka Pharmaceutical Development \& Commercialization, Inc., Princeton, NJ, USA 